\title{
Molecular Dynamics Study of Dipalmitoylphosphatidylcholine Lipid Layer Self-Assembly onto a Single-Walled Carbon Nanotube
}

\author{
Hongming Wang, Servaas Michielssens, Samuel L. C. Moors, and Arnout Ceulemans（ $\varangle)$ \\ Laboratory of Quantum Chemistry, Department of Chemistry and INPAC Institute for Nanoscale Physics and Chemistry, Katholieke \\ Universiteit Leuven, Belgium \\ Received: 28 August 2009 / Revised: 19 October 2009 / Accepted: 19 October 2009 \\ OTsinghua University Press and Springer-Verlag 2009. This article is published with open access at Springerlink.com
}

\begin{abstract}
Single-walled carbon nanotubes (SWNTs) are possible nano-injectors and delivery vehicles of molecular probes and drugs into cells. In order to explore the interaction between lipid membranes and carbon nanotubes, we investigate the binding mechanism of dipalmitoylphosphatidylcholine (DPPC) with SWNTs by molecular dynamics. In low concentration range simulations, the DPPC molecules form a supramolecular two-layered cylindrical structure wrapped around the carbon nanotube surface. The hydrophobic part of DPPC is adsorbed on the surface of the nanotube, and the hydrophilic top is oriented towards the aqueous phase. For higher concentration ranges, the DPPC molecules are found to form a supramolecular multi-layered structure wrapped around the carbon nanotube surface. At the saturation point a membrane-like structure is self-assembled with a width of $41.4 \AA$, which is slightly larger than the width of a cell membrane. Our study sheds light on the existing conflicting simulation data on adsorption of single-chained phospholipids.
\end{abstract}

\section{KEYWORDS}

Cell membrane, molecular dynamics, carbon nanotubes, self-assembly, nano-injector

\section{Introduction}

Single-walled carbon nanotubes (SWNTs) offer unique opportunities for chemical and biological sensing $[1,2]$ and have great potential applications in electronics, optics, mechanics, and thermal transportation [3, 4]. The interaction of biomolecules with SWNTs has generated a great deal of interest in the past few years. Chen et al. reported a translocation of multi-walled carbon nanotubes across a breast cancer cell [5] and demonstrated the capability of the tube to act as a nano-injector into the cell cytoplasm without cell membrane damage. On the other hand, an SWNT inside a cell was reported to localize within the nucleus, causing cell mortality in a dose-dependent manner [6]. Several studies indicate that functionalized SWNTs can be spontaneously inserted into a lipid bilayer [7-10]. Klein et al. reported that a generic nanotube functionalized with hydrophilic termini is assisted in crossing the membrane core by transleaflet lipid flips [11, 12]. Wallace and Sansom used coarse-grained molecular dynamics to simulate the penetration of dipalmitoyl phosphatidylcholine (DPPC) bilayers by SWNTs: the

Address correspondence to Arnout.Ceulemans@chem.kuleuven.be 
degree of lipid lining of the inner surface was found to be strongly dependent upon the tube penetration velocity, with fewer lipids extracted from the bilayer at higher rates [13]. There are thus promising perspectives for the use of nanotubes and nanopores in molecular sequencing and selective molecular transport. It is obvious that structural reliability and low reactivity are necessary for this kind of nanoinjector to deliver drugs into cells through an artificial transfer channel. So, it is very important to know the mechanism of interaction between cell membranes and nanotubes.

The transporter property of carbon nanotubes has initiated many studies on the interaction and binding mechanism between carbon nanotubes and the lipid components of the cell membrane. The observation of band gap fluorescence of individual SWNTs in an aqueous dispersion of sodium dodecyl sulphate (SDS) led O'Connell et al. to conclude that the nanotube was encased in a cylindrical micelle with the surfactant molecule oriented perpendicularly to the surface of the tube [14]. However, using transmission electron microscopy, Richard et al. were able to visualize clear striations in the lipid coating on an SWNT, which were thought to be due to rolled-up half-cylinder lipid structures on the nanotube surface [15]. McEuen et al. reported that lipid molecules can diffuse freely across the tube [16] and Wu et al. examined the coating of SWNTs with single and double-chained phospholipids [17].

To elucidate the nature of the supramolecular assembly between single-chained lysophosphatidylcholine (LPC) and carbon nanotubes, Qiao and Ke performed 24 ns atomistic molecular dynamics (MD) simulations of the interaction of LPC with a $(18,0)$ SWNT and found that the lipids were predominately aligned with the tube axis as opposed to the hemi-micelle mode [18]. however, a later study by Wallace and Sansom, using coarsegrained MD, proved to be at odds with this result, yielding a structure which was more consistent with the earlier cylindrical detergent encapsulation mode [19]. In view of this discrepancy, the interaction mechanism of lipids and SWNTs remains unclear. In order to reach a better understanding of the binding mechanism of cell membrane lipids with SWNTs, we present a molecular dynamics simulation study involving the double-chained DPPC. In contrast to LPC, such lipids are believed to be curvophobic and to induce a bilayer packing [20].

\section{Computational methods}

We performed classical molecular dynamics simulations to elucidate the binding of DPPC on an SWNT. Initially, the dimensions of the simulation box were set at $10 \mathrm{~nm} \times 10 \mathrm{~nm} \times 10 \mathrm{~nm}$. The initial simulation system consisted of the SWNT fixed along the $z$-axis at the center of the box, 46 DPPC molecules and 30577 water molecules. We chose a $(18,0)$ zigzag SWNT, $13.9 \AA$ in diameter and $96.5 \AA$ in length containing 1440 carbon atoms. Since the nature of the interactions between carbon nanotubes and lipids has been reported to be strongly dependent on the concentration of lipid [19], we also performed simulations with higher concentrations. In fact, it has been reported that the binding mechanism of lipid adsorption onto SWNTs not only depends upon the concentration of lipid, but also on the weight ratio of lipid to SWNT when experimental mass ratios were varied from 1:1 to 10:1, and concentrations of lipid were in the range $1 \%$ to $10 \%$. The initial box filled with 46 DPPC molecules yields a mass ratio of DPPC to SWNT of about 1.95:1. To cover the estimated concentration range, we also performed simulations for higher concentrations with 88 and 173 DPPC molecules in a box of the same size, with 28529 and 24799 water molecules, respectively. In all these simulations, initially the DPPC molecules were distributed roughly uniformly over the box.

Water was simulated with the simple point charge (SPC) model [21]. Periodic boundary conditions were applied in all three directions. The electrostatic interactions were computed using the particle mesh Ewald method [22] with a fast Fourier transform (FFT) grid spacing of $0.11 \mathrm{~nm}$. The time step size was $2 \mathrm{fs}$. Based on previous work, the SWNT atoms were modeled as uncharged Lennard-Jones particles using $\mathrm{sp}^{2}$ carbon parameters [23, 24] with a cross section of $\sigma_{\mathrm{CC}}=0.365 \mathrm{~nm}$, and a depth of the potential well of $\varepsilon_{\mathrm{CC}}=0.465 \mathrm{~kJ} / \mathrm{mol}$. For the DPPC molecule, we used Berger's parameters [25]. For all the bond 
lengths, valence angles, and improper dihedrals, as well as for dihedral angles in the head-group region, the standard parameters of the GROMOS force field were used. For the hydrocarbon chains the Ryckaert-Bellemans potential was used. For more details about the parametrization see the reports [26, 27]. Computer simulation of the cell membrane is determined strongly by the available parameter sets for the lipid. To study this effect we also performed an additional simulation of the same system of 46 DPPC molecules using the GROMOS force field based on the last GROMOS96 parameter set termed ffG53a6 for DPPC [28]. A Berendsen thermostat with a coupling constant of 0.1 ps was applied in all simulations [29]. The reference temperature was set to $300 \mathrm{~K}$. Pressure coupling was applied isotropically, also using the Berendsen scheme with a coupling constant of 1.0 ps. The reference pressure was 1 bar in all directions. Bond lengths were constrained with the LINCS algorithm [30]. An energy minimization of the system using the method of steepest descent and a long run with a leapfrog stochastic dynamics integrator was carried out for the equilibration at 300 $\mathrm{K}$ with a friction constant of $0.5 \mathrm{~ns}^{-1}$. After energy minimization, a simulation with position restraints on DPPC molecules was carried out at $300 \mathrm{~K}$ for 400 ps. All the simulations were performed using the Gromacs MD simulation package at constant temperature (323 K) and pressure (1 bar) [31, 32] .

\section{Results and discussion}

\subsection{Low concentration range}

Cell membranes are self-assembled bilayers of phospholipids. Using molecular dynamics, Kasson and Pande have modeled the creation of bilayer gaps through the reorientation of lipids at bilayer edges [33]. Near these edges a rapid reorientation and movement of phospholipids was observed. The rapid local rearrangement of lipids during membrane fusion may also facilitate the formation of fusion intermediates, thought key to the infection cycle of viruses. The alkyl chain of DPPC is hydrophobic, whereas the top of the phospholipid is hydrophilic [34]. The graphene surface of an SWNT is also hydrophobic. We therefore expect that the polar top of DPPC would not readily adsorb onto the external surface of an SWNT, as compared to the alkyl chain. From our simulation at low concentration, we observe that during the first stage of the rearrangement $(0$ $1.2 \mathrm{~ns})$, DPPC molecules cluster into small micellelike aggregates, and a ring starts to be formed around the SWNT from 0 to 1.2 ns (Fig. 1(b)) that can reduce the exposed solvent-accessible surface of the hydrophobic detergent tails. Gradually, both free DPPC molecules and DPPC clusters adsorb from the solution onto the SWNT; the process is shown in Fig. 1. Apparently, the formation of micelle-like structures and the adsorption of DPPC on SWNTs are almost concerted. The important jump occurs from 1 to 3.0 ns, and corresponds to the adhesion of a large lipid cluster to form a structure with two concentric layers. At that time there are about 40 DPPC molecules absorbed on the SWNT. Eventually no clusters of DPPC molecules remain in solution, indicating that the coating may be saturated.

The adsorption of the lipid layers is in fact very fast; it only takes about 2 ns. Figures 1(d) and 1(e) present front and side views of the simulation system at $t=15 \mathrm{~ns}$. We find that the DPPC chains are wrapped around the tube, mostly with a wrapping angle of $20^{\circ}-40^{\circ}$ with respect to the SWNT long axis [19]. Our result is in very good agreement with the findings at low concentration by Wallace and Sansom [19], where LPC wraps around the SWNT at low detergent concentration with an average wrapping angle of $\sim 48^{\circ}$. The hydrophobic part of the DPPC is adsorbed on the surface of the SWNT, and the hydrophilic top is oriented towards the aqueous phase, forming two cylindrical layers on the surface of the SWNT. Such a structure protects the hydrophobic interior of DPPC and the surface of the SWNT from exposure to water while keeping the hydrophilic head groups in contact with the solution. The longitudinal ordering which we observe is rather similar to the results of Qiao and Ke for the singlechained LPC [18]. While for LPC the lipid tails are mostly stretched along the tubes axis, we find that the DPPC molecules can also be wrapped around the tube. The polar heads cluster at regular intervals of about $2.5 \mathrm{~nm}$, which is more crowded than in the 


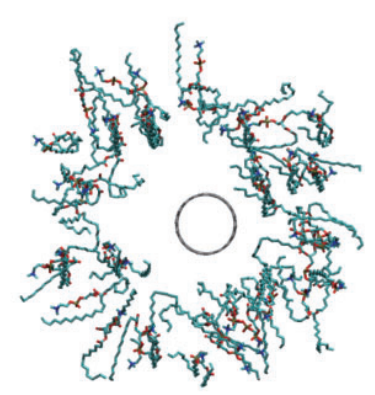

(a)

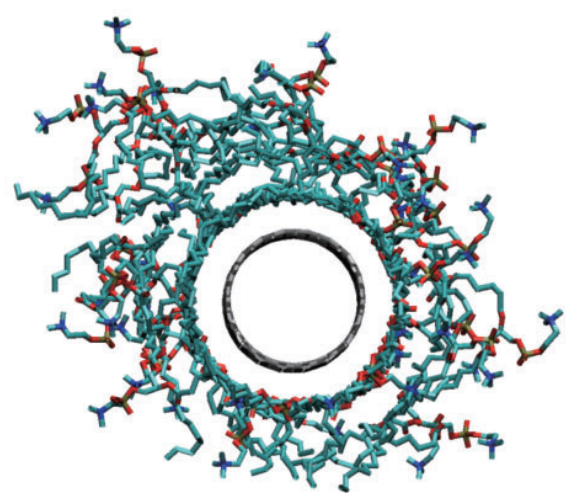

(d)

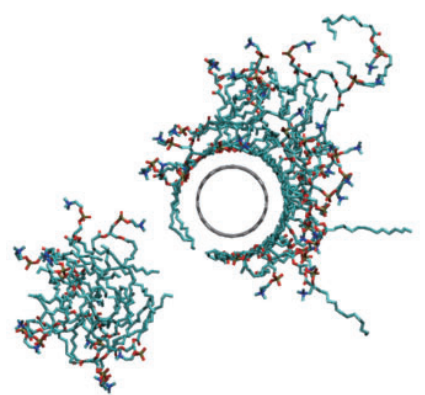

(b)

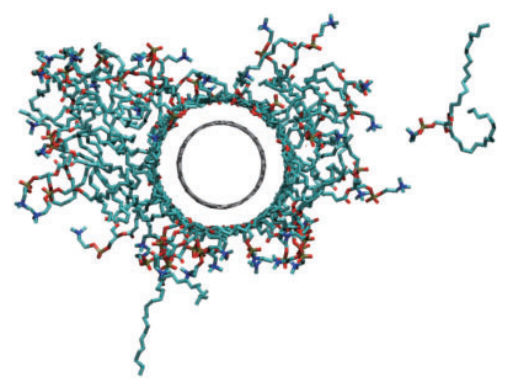

(c)

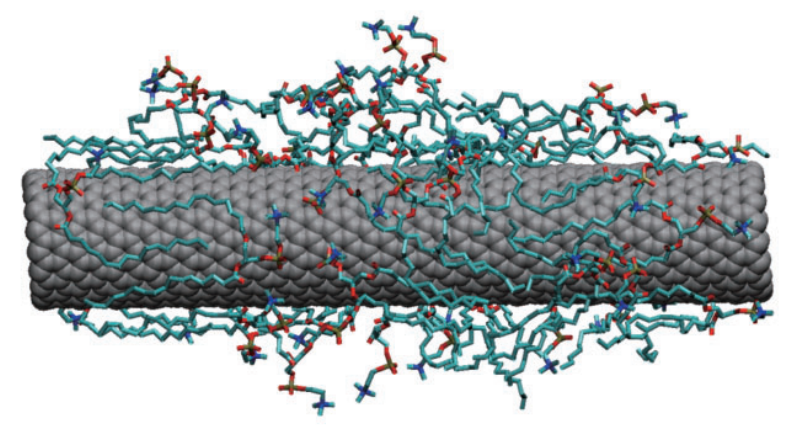

(e)

Figure 1 The self-assembly of DPPC and an SWNT for 46 DPPC molecules. (a), (b), (c), and (d) show radial views of the SWNT-DPPC simulation system ((a): 0 ns; (b): 1.2 ns; (c): 3 ns; (d): $15 \mathrm{~ns})$. (e) Side view of the simulation system configuration at 15 ns

simulation of Qiao and Ke [18]; this may be due to the smaller size of the SWNT which we use in this simulation. The novel feature is the clear transversal ordering in a two-stack structure, as shown in Fig. 2. The distance between the surface of the nanotube and the first ring is $4.06 \AA$ and the distance between the first and the second ring is $4.4 \AA$ (Fig. 2(a)). The latter value is the same as the distance between two chains of lipid in the cell membrane. For the first layer, we find that the entire length of the hydrophobic tail of DPPC is almost parallel to the surface of the SWNT. This result confirms the earlier expectation that double-chained phospholipids pack in concentric double layers [16]. However, we did not find evidence for their alleged "curvophobia". The radial distribution function (RDF) of all DPPC atoms around the SWNT as a function of radial distance between the axis of the SWNT and the DPPC atoms is shown in Fig. 2(a). Two discrete adsorption peaks can be observed at 11.01 and $15.41 \AA$, which show that there are clearly two layers around the SWNT. The first layer is due to the interfacial van der Waals interaction between the DPPC chain and the SWNT. The second layer is further supporting the high molecular density in the first layer. The same structure has been observed in short and long polymer molecules wrapped around SWNTs in previous simulation studies. Wei reported an MD simulation of the conformation of linear polymer molecules at an SWNT interface which showed that the polymer molecules preferentially adopt certain conformations on the SWNT surface to form a twolayer structure [35].

\subsection{High concentration range}

Both Qiao and Ke [18] and Wallace and Sansom [19] reported that the mechanism of adsorption of LPC onto SWNTs is dependent upon LPC concentration. Low concentrations of detergent lead to LPC tails adsorbing in between the thread lines of the SWNT. In order to clarify the concentration effect for the case of DPPC, we have repeated the simulations for two higher concentrations of DPPC, with 88 and 173 DPPC molecules, keeping the same box size.

For the case of 88 DPPC molecules, after 15 ns, we again observe the formation of two concentric layers, 


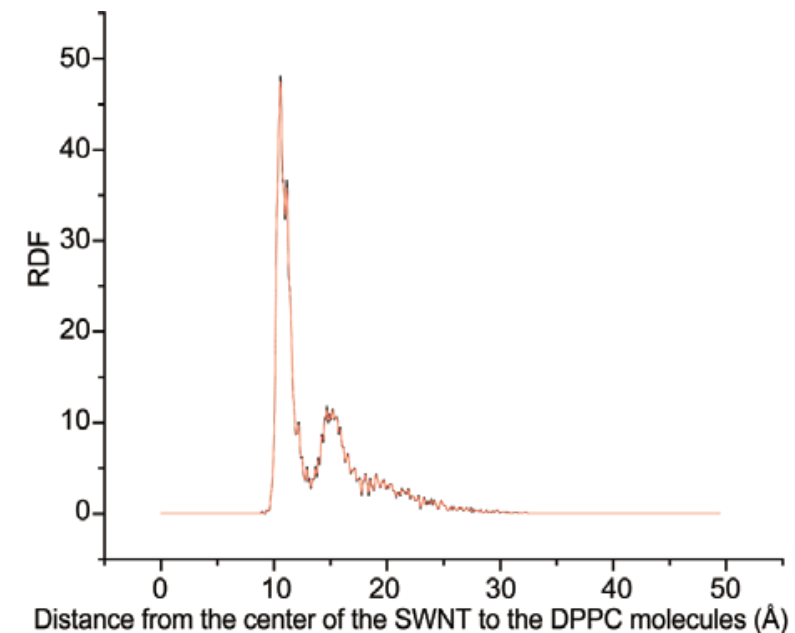

(a)

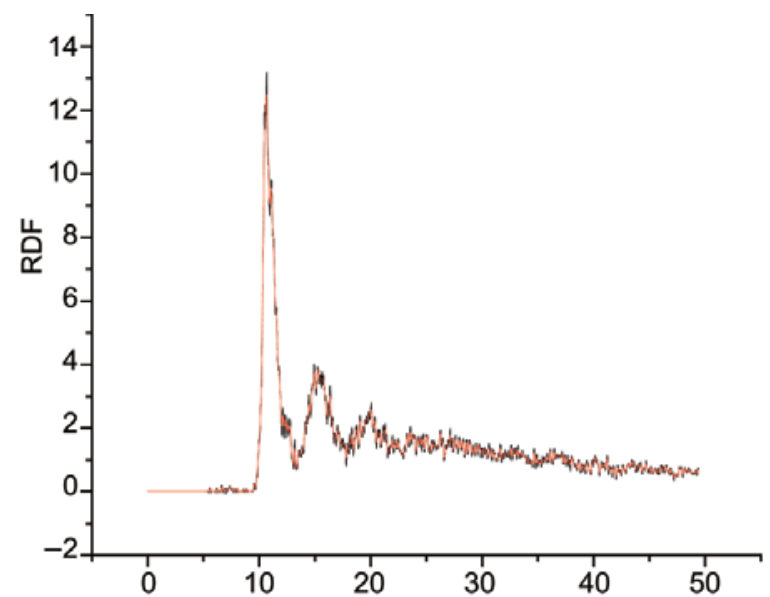

Distance from the center of the SWNT to the DPPC molecules $(\AA)$

(c)

Figure 2 RDF as a function of distance between the center of the SWNT and the DPPC atoms ((a) 46 DPPCs, (b) 88 DPPCs, and (c)173 DPPCs) (distances in $\AA$ )

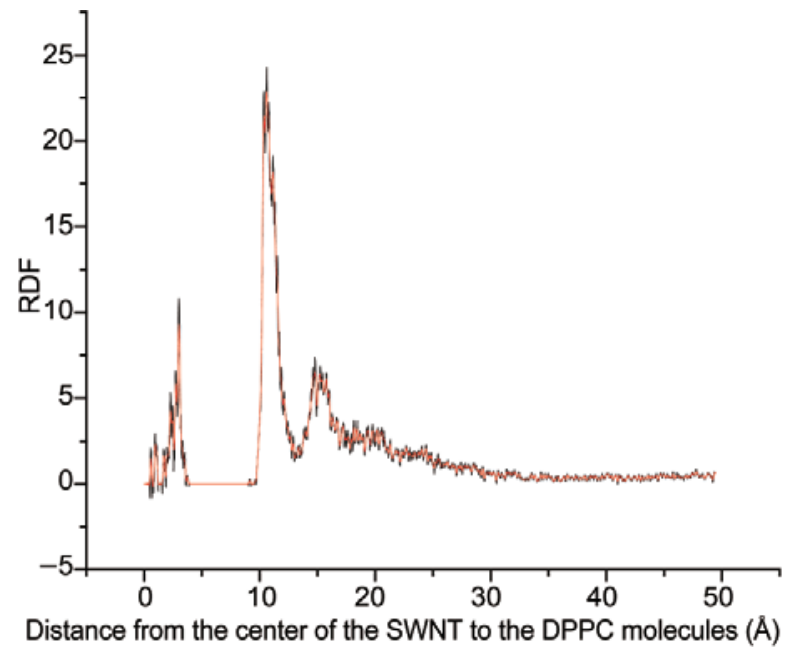

(b)

as shown in Fig. 3. For the first layer, the distance to the wall of the SWNT is about $4.3 \AA$, which is almost the same as in the previous low concentration case of 46 DPPCs. The RDF of the DPPC molecules around the SWNT as a function of radial distance from the center of the SWNT, as illustrated in Fig. 2(b), displays two peaks at 11.03 and $15.43 \AA$, which confirm the existence of two adsorption layers. Compared to the previous case, fewer DPPC molecules have the whole length of their hydrophobic tail in contact with the first layer. Moreover, the DPPC molecules undergo a rotation such that the contact angle between the tube wall and the lipid is larger than is the case at low concentration. Presumably, this transition enables more DPPC molecules to be incorporated into the DPPC-SWNT complex, thereby minimizing

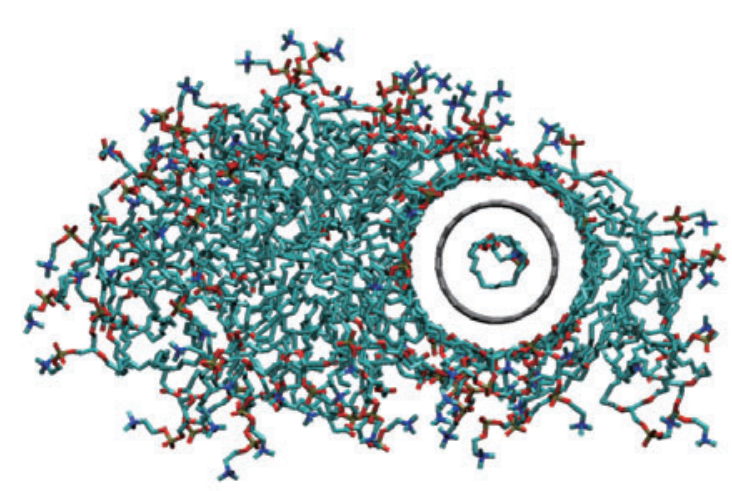

(a)

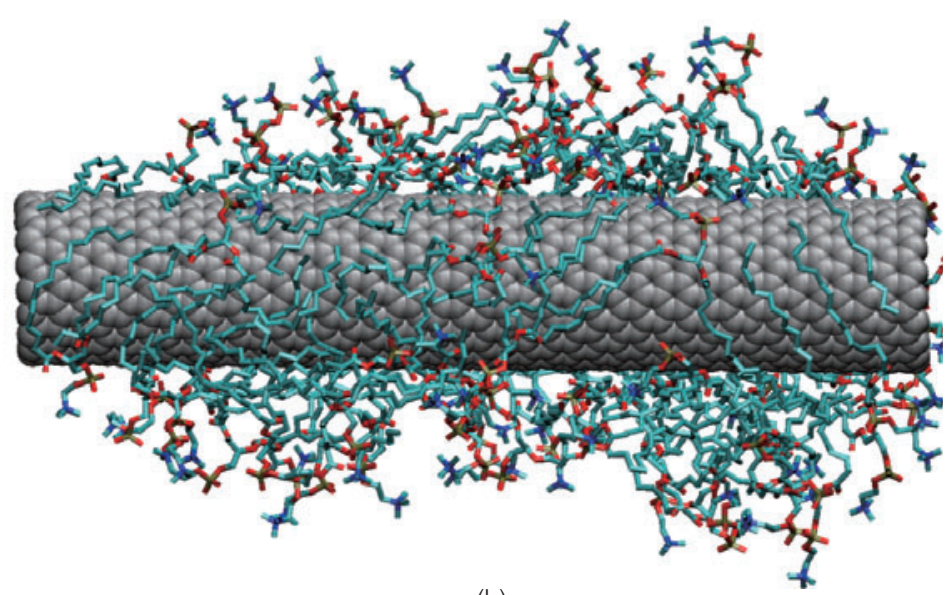

(b)

Figure 3 The self-assembly of 88 DPPC molecules on an SWNT: (a) side view of the simulated system at 15 ns; (b) front view of the simulated system at $15 \mathrm{~ns}$

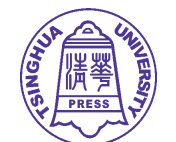


exposure of the hydrophobic SWNT and the DPPC hydrophobic tail to the aqueous environment. In fact, at this concentration, the lipid chain of DPPC in the first layer is around the SWNT and the entire length of the hydrophobic tail of DPPC is nearly parallel with the surface of the SWNT. Undoubtedly, this contact mode must be attributed to the interfacial van der Waals interaction between the DPPC chain and the SWNT. The hydrophilic top of DPPC is mainly oriented towards the aqueous phase, and makes a large angle with the $z$-axis of the SWNT. The adsorption mechanism observed at this concentration is in good agreement with the cylindrical detergent encapsulation model, and partly consistent with the result for high concentration reported by Wallace and Sansom [19].

For an even higher concentration of 173 DPPC molecules the lipid molecules have a strong tendency to form small micelle-like aggregates. So, the rate of adsorption is lower than that at low concentrations, and it takes approximately 6 ns before the adsorption is completed. In an initial time span of $2 \mathrm{~ns}$, individual DPPC molecules rapidly fuse to form small micellelike aggregates of several DPPC molecules each, thereby reducing the exposed solvent accessible surface (SAS) [36] of the hydrophobic detergent tails. Consequently, lipids that bridge the micelles lead to the creation of larger, lamellar aggregates as shown in Fig. 4(a). The lamellar structures are in essence curved layers around the SWNT. Within $\sim 5 \mathrm{~ns}$, only three isolated detergent micelles remain, which is consistent with a reduction in the average SAS per detergent from 10.6 to $5.2 \mathrm{~nm}^{2}$, primarily as a result of burial of DPPC tails. Figure 5 shows the variation of detergent SAS per detergent molecule over time. In the first $1 \mathrm{~ns}$, the average SAS of DPPC molecules decreases very fast from 10.6 to $6.7 \mathrm{~nm}^{2}$ for the total molecule, from 6.5 to $4.2 \mathrm{~nm}^{2}$ for the tail and from 4.4 to $2.6 \mathrm{~nm}^{2}$ for the head. The average SAS of DPPC converges towards the end of the simulation after 15.6 ns to almost 4.3 (total), 2.5 (tail), and 1.6 (head) $\mathrm{nm}^{2}$, which suggests that the SWNT-DPPC system has reached a stable minimum. Water-bounded edges appear to attain stability by reorienting to present a polar surface at the lipid-water interface, protecting the hydrophobic tail groups.
Figures 4(c), 4(d), and 4(e) give the end and side views of equilibrated DPPC-SWNT complexes with 173 DPPC molecules. Very interestingly, as can be see from Figs. 4(c) and 4(e), a membrane-like structure is established, in which the SWNT occupies the center so as to form a nano-injector on the cell membrane, that bears similarity to the experimental result reported by Chen et al. [5]. In this structure, the DPPC forms a large oblate micelle, and the tube is oriented across this. The structure is analogous to that observed in MD simulations of SWNT penetration in a bilayer membrane, as recently reported by Wallace and Sansom [13]. In this paper, the authors used coarse-grained molecular dynamic to simulate the penetration of DPPC bilayers by SWNTs and found that the SWNTs can be used as delivery system. The difference between this coarse-grained study and our simulation is that Wallace and Sansom pulled the SWNT through the membrane at a constant velocity. Hence, both the orientation and forces in this study are subject to bias, which is absent in our study. From the side view (Fig. 4(d)), this membrane is a gapped bilayer, and the micellization at gap boundaries is clearly visible. This introduces a large curvature of the cell membrane which can help to minimize the edge energy [37]. Our simulations of the gapped bilayer show a very slight increase in bilayer width near the edges. This "bulging" of the bilayer ends may help to solve the packing problem created by micellization. In fact, such a cell membrane structure can protect the hydrophobic interior of the bilayer from exposure to water. The width of the membrane is about $41.4 \AA$, which is slightly larger than the width of a cell membrane. The presence of gaps in this kind of membrane will lead to a slight increase in bilayer width. Kasson and Pande [33] presented a model for gap formation in which the width of the lipid bilayer indeed increases slightly. For a dimyristoylphosphatidylcholine (DMPC) bilayer, the increase in width is almost $3.7 \AA$ A. Figure 2(c) shows the RDF for the high-concentration limit as a function of distance between the SWNT centers and the DPPC molecules. Three main peaks are clearly visible at 11.31, 15.91, and $20.52 \AA$, which indicate that at least three adsorption layers were formed. This result is very different from the micelle-like conformation 


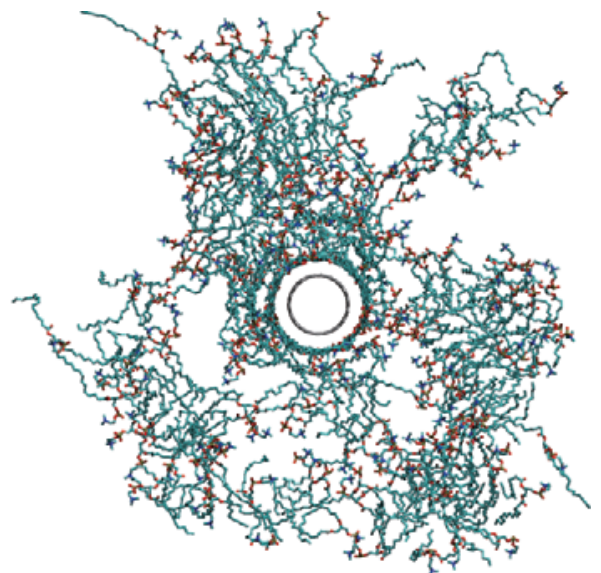

(a)

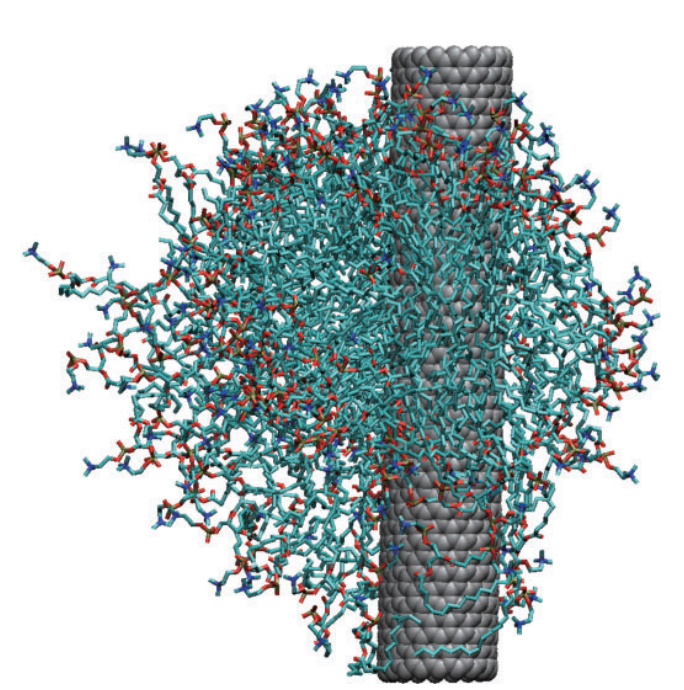

(d)

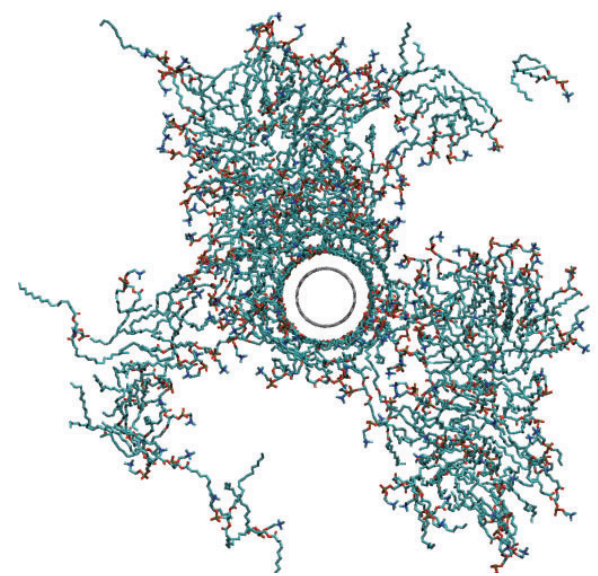

(b)

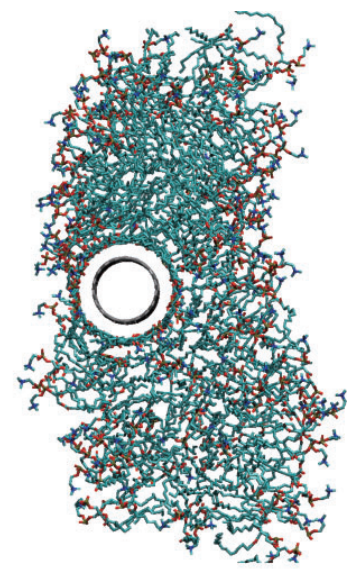

(c)

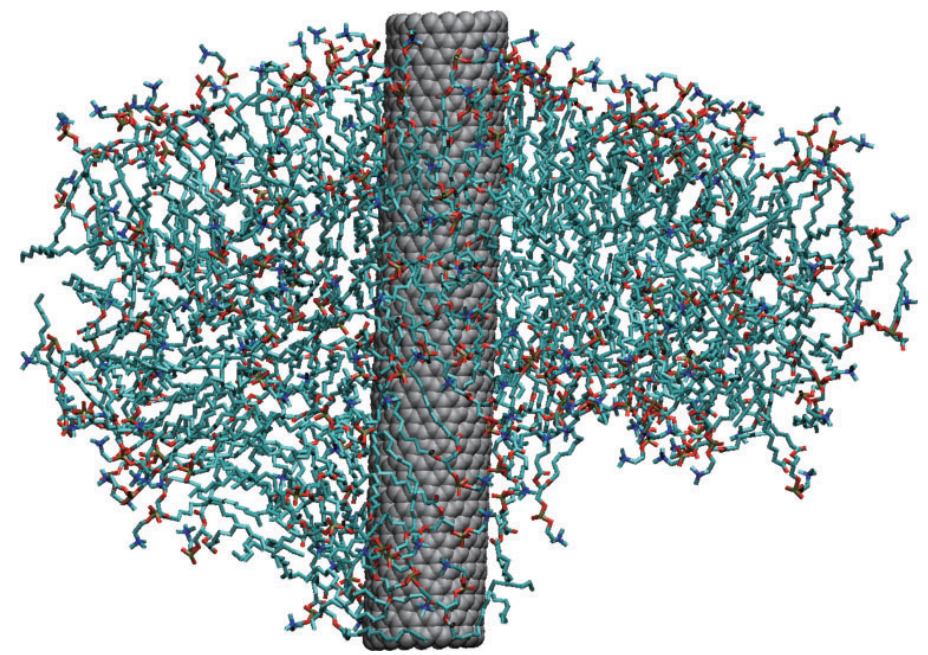

(e)

Figure 4 The self-assembly of DPPC on an SWNT for 173 DPPC molecules. (a) and (b) show radial views of the SWNT-DPPC simulation system ((a): 1 ns; (b): 3 ns). (c) Side view of the simulation system configuration at 15 ns. (d) and (e): Front views of the simulation system configuration at $t=15 \mathrm{~ns}$

which was reported for high LPC concentration [19].

Why is the structure of SWNT-LPC so different from the SWNT-DPPC aggregate at high concentration? From a structural point of view, LPC molecules may be regarded as an extreme case of the asymmetric phosphatidylcholine system in which the entire sn-2 acyl chain (the second chain) of DPPC is substituted by a hydrogen atom. The wedge shape of LPC molecules strongly favors the formation of micelles over bilayers [33]. In contrast, in DPPC there are two chains, sn- 1 and sn-2, which yield a larger area for the hydrophobic tail as compared to the area of the hydrophilic top. This means that it is impossible to pack the non-polar tails in a micellar surface formed by the polar tops. Lu et al. [38] have reported the effect of LPC on the behavior and structure of phosphatidylcholine liposomes: a high concentration of LPC additives will convert the DPPC bilayer to a micelle, which confirms that LPC itself tends to micellization, while DPPC prefers to form a membrane. This explains very clearly why a micellar structure was obtained by the selfassembly of LPC on SWNTs in the simulation study of Wallace and Sansom [19], while in our study at high concentration of DPPC on SWNTs we observe the gradual development of a cell membrane.

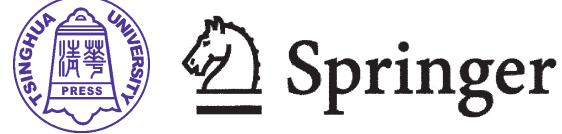




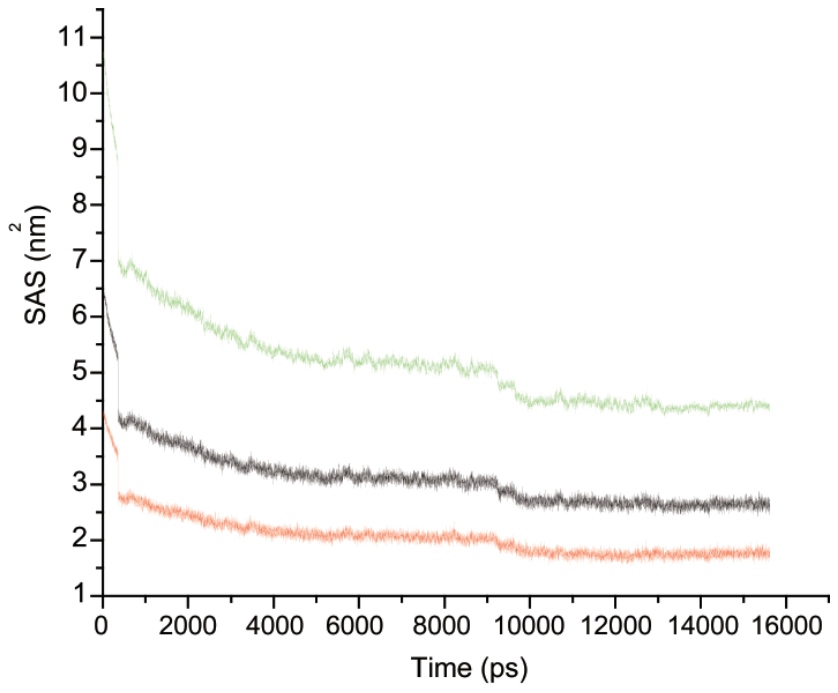

Figure 5 Variation of SAS area per detergent molecule over time, for all detergents of the main detergent aggregate with a concentration of 173 DPPC molecules. The SAS per detergent is shown for the entire detergent molecule (top green line), for the hydrocarbon chain tail (central black line) and for the polar head group regions (bottom red line)

\subsection{Influence of the force field parameters}

As a further check we have repeated the simulations for the low-concentration case with 46 DPPC molecules using the GROMOS96 force field parameters for DPPC [28]. The result from the simulation is in good agreement with the previous results using Berger's parameters and confirms that the DPPC tails wrap around the SWNT to form two concentric layers as shown in Figs. 6(a) and 6(b). This observation also is in line with a previous MD investigation by Wei [35]. Such a structure protects the hydrophobic interior of DPPC and the surface of SWNTs from exposure to water while keeping the hydrophilic head groups in contact with the solution. After $5 \mathrm{~ns}$, no clusters of DPPC molecules remain in solution, indicating that all DPPC molecules were adsorbed on the SWNT. Figure 6(c) gives the RDF of the DPPC molecules, as obtained with the alternative parameters. Two adsorption layers are found at 11.31 and $15.73 \AA$. These values are about $2 \%$ larger than the ones obtained by Berger's parametrization, indicating that the alternative parameters very slightly tend to reduce the van der Waals attraction between DPPC and the SWNT. Overall the agreement is very good, which indicates that the parametrization is reliable.

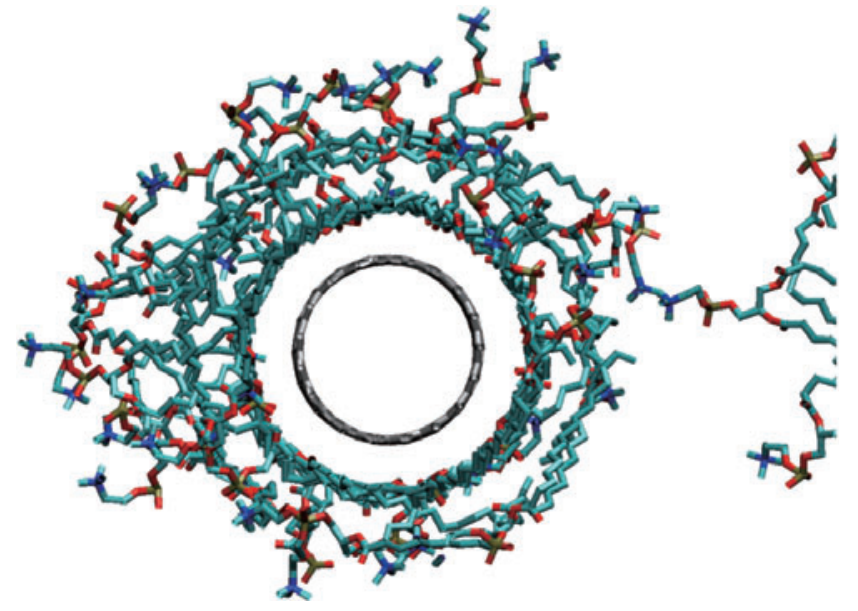

(a)

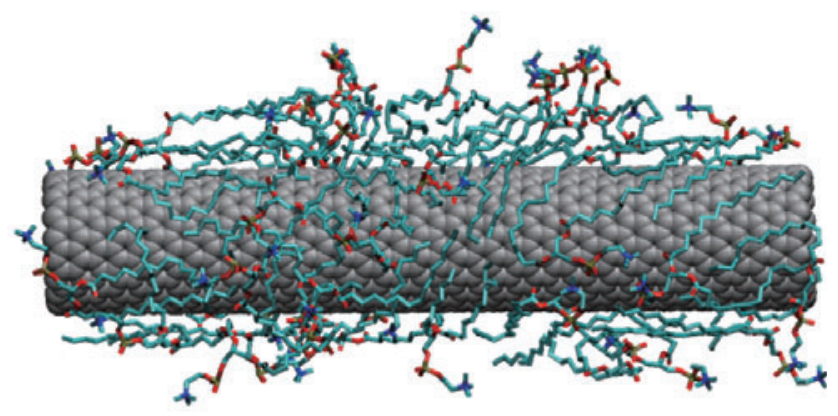

(b)

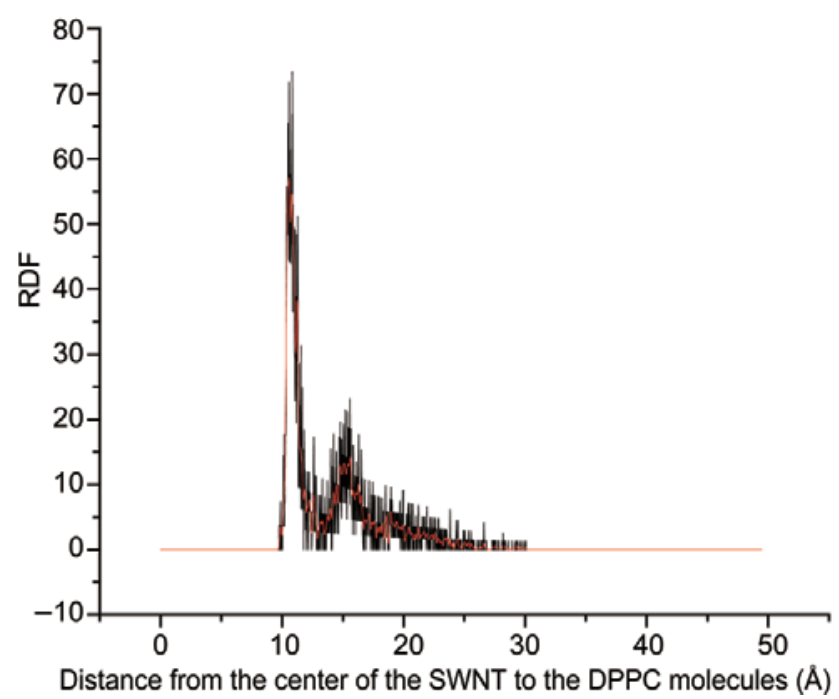

(c)

Figure 6 The self-assembly of DPPC and an SWNT for 46 DPPC molecules using GROMOS96 parameters [28] for DPPC: (a) side view of the simulation system configuration at $15 \mathrm{~ns}$; (b) front view of the simulation system configuration at $15 \mathrm{~ns}$; (c) RDF as a function of distance between the axis of the SWNT and the center of mass of each DPPC molecule 


\section{Conclusions}

Our simulations clearly reveal that the interaction between SWNTs and DPPC is concentrationdependent. For low concentrations, the DPPC molecules form a supramolecular two-layer cylindrical structure wrapped around the SWNT surface. The hydrophobic part of the DPPC is adsorbed on the surface of the SWNT, and the hydrophilic top is oriented towards the aqueous phase. Our results confirm that nanotubes provide a suitable template for molecular self-organization, which is characterized by small tilting angles due to hydrogen bonding. However, for high concentration, the DPPC molecules form a supramolecular multilayered structure wrapped around the SWNT surface. The first layer is due to van der Waals interactions between DPPC chains and the SWNT. The second layer further supports the high molecular density in the first layer. A membrane-like structure develops, with a width of $41.4 \AA$. This result is typical for DPPC, and is at variance with the micellar characteristics of LPC simulations. This result suggests that SWNTs may be promising nanotools for the delivery of cargo into a cell. The experimental observations of DPPC coatings on nanotubes with regular striations are in line with the side-on modes of aggregation suggested by our atomistic simulations.

\section{Acknowledgements}

We thank the Flemish government for financial support through the Concerted Action Scheme. H. W. is indebted to INPAC for a postdoctoral grant. S. M. is the recipient of a doctoral grant from the Flemish Science Foundation (FWO).

\section{References}

[1] Star, A.; Gabriel, J. P.; Bradley, K.; Grüne, G. Electronic detection of specific protein binding using nanotube FET devices. Nano Lett. 2003, 3, 459-463.

[2] Besteman, K.; Lee, J. -O.; Wiertz, F. G. M.; Heering, H. A.; Dekker, C. Enzyme-coated carbon nanotubes as singlemolecule biosensors. Nano Lett. 2003, 3, 727-730.

[3] Chen, R. J.; Zhang, Y. G.; Wang, D. W.; Dai, H. J.
Noncovalent sidewall functionalization of single-walled carbon nanotubes for protein immobilization. J. Am. Chem. Soc. 2001, 123, 3838-3839.

[4] Kam, N. W. S.; Jessop, T. C.; Wender, P. A.; Dai, H. Nanotube molecular transporters internalization of carbon nanotube-protein conjugates into mammalian cells. J. Am. Chem. Soc. 2004, 126, 6850-6851.

[5] Chen, X.; Kis, A.; Zettl, A.; Bertozzi, C. R. A cell nanoinjector based on carbon nanotubes. Proc. Natl. Acad. Sci. USA 2007, 104, 8218-8222.

[6] Porter, A. E.; Gass, M.; Muller, K.; Skepper, J. N.; Midgley, P. A.; Welland, M. Direct imaging of single-walled carbon nanotubes in cells. Nat. Nanotechnol. 2007, 2, 713-717.

[7] Vakarelski, I. U.; Brown, S. C.; Higashitani, K.; Moudgil, B. M. Penetration of living cell membranes with fortified carbon nanotube tips. Langmuir 2007, 23, 1089310896.

[8] Bianco, A.; Hoebeke, J.; Godefroy, S.; Chaloin, O.; Pantarotto, D.; Briand, J.; Muller, S.; Prato, M.; Partidos, C. D. Cationic carbon nanotubes bind to CpG oligodeoxynucleotides and enhance their immunostimulatory properties. J. Am. Chem. Soc. 2005, 127, 58-59.

[9] Cherukuri, P.; Bachilo, S. M.; Litovsky, S. H.; Weisman, R. B. Near-infrared fluorescence microscopy of single-walled carbon nanotubes in phagocytic cells. J. Am. Chem. Soc. 2004, 126, 15638-15639.

[10] Lu, Q.; Moore, J. M.; Huang, G.; Mount, A. S.; Rao, A. M.; Larcom, L. L.; Ke, P. C. RNA polymer translocation with single-walled carbon nanotubes. Nano Lett. 2004, 4, 2473-2477.

[11] Lopez, C. F.; Nielsen, S. O.; Moore, P. B.; Klein, M. L. Understanding nature's design for a nanosyringe. Proc. Natl. Acad. Sci. USA 2007, 101, 4431-4434.

[12] Lopez, C. F.; Nielsen, S. O.; Ensing, B.; Moore, P. B.; Klein, M. L. Structure and dynamics of model pore insertion into a membrane. Biophys. J. 2005, 88, 3083-3094.

[13] Wallace, E. J.; Sansom, M. S. P. Blocking of carbon nanotube based nanoinjectors by lipids: A simulation study. Nano Lett. 2008, 8, 2751-2756.

[14] O'Connell, M. J.; Bachilo, S. M.; Huffman, C. B.; Moore, V. C.; Strano, M. S.; Haroz, E. H.; Rialon, K. L.; Boul, P. J.; Noon, W. H.; Kittrell, C.; Ma, J.; Hauge, R. H.; Weisman, R. B.; Smalley, R. E. Band gap fluorescence from individual single-walled carbon nanotubes. Science 2002, 297, 593-596.

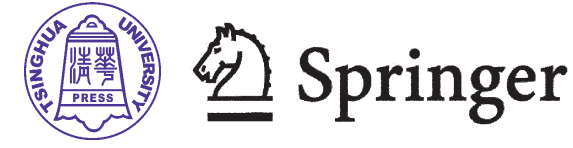


[15] Richard, C.; Balavoine, F.; Schultz, P.; Ebbesen, T. W.; Mioskowski, C. Supramolecular self-assembly of lipid derivatives on carbon nanotubes. Science 2003, 300, 775-778.

[16] Zhou, X. J.; Moran-Mirabal, J. M.; Craighead, H. G.; McEuen, P. L. Supported lipid bilayer/carbon nanotube hybrids. Nat. Nanotechnol. 2007, 2, 185-190.

[17] Wu, Y.; Hudson, J. S.; Lu, Q.; Moore, J. M.; Mount, A. S.; Rao, A. M.; Alexov, E.; Ke, P. C. Coating single-walled carbon nanotubes with phospholipids. J. Phys. Chem. B. 2006, 110, 2475-2478.

[18] Qiao, R.; Ke, P. C. Lipid-carbon nanotube self-assembly in aqueous solution. J. Am. Chem. Soc. 2006, 128, 1365613657.

[19] Wallace, E. J.; Sansom, M. S. P. Carbon nanotube/ detergent interactions via coarse-grained molecular dynamics. Nano Lett. 2007, 7, 1923-1928.

[20] Smondyrev, A.; Berkowitz, M. L. Molecular dynamics study of Sn-1 and Sn-2 chain conformations in dipalmitoylphosphatidylcholine membranes. J. Chem. Phys. 1999, 110, 3981-3985.

[21] Berendsen, H. J. C. J. P.; Postma, M.; van Gunsteren, W. F.; Hermans, J. In: Proceedings of the Fourteenth Jerusalem Symposium on Quantum Chemistry and Biochemistry, Jerusalem, Israel, April 13-16, Pullman, B., Ed.; Kluwer: Reidel, Dordrecht, 1981; p. 331.

[22] Essman, U.; Perela, L.; Berkowitz, M. L.; Darden, T.; Lee, H.; Pedersen, L. G. A smooth particle mesh Ewald method. J. Chem. Phys. 1995, 103, 8577-8593.

[23] Rossetti, G.; Magistrato, A.; Pastore, A.; Persichetti, F.; Carloni, P. Structural properties of polyglutamine aggregates investigated via molecular dynamics simulations. J. Phys. Chem. B 2008, 112, 16843-16850.

[24] Shao, Q.; Huang, L.; Zhou, J.; Lu, L.; Zhang, L.; Lu, X.; Jiang, S.; Gubbins, K. E.; Zhu, Y.; Shen, W. Molecular dynamics study on diameter effect in structure of ethanol molecules confined in single-walled carbon nanotubes. J. Phys. Chem. C 2007, 111, 15677-15685.

[25] Berger, O.; Edholm, Olle.; Jahnig, F. Molecular dynamics simulations of a fluid bilayer of dipalmitoylphosphatidylc holine at full hydration, constant pressure, and constant temperature. Biophys. J. 1997, 72, 2002-2013.

[26] Egbert, E.; Marrink, S. J.; Berends, H. J. C. Molecular dynamics simulation of a phospholipid membrane. Eur. Biophys. J. 1994, 22, 423-436.
[27] Ryckaert, J. P.; Bellemans, A. Molecular dynamics of liquid $n$-butane near its boiling point. Chem. Phys. Lett. 1975, 30, 123-125.

[28] Oostenbrink, C.; Villa, A.; Mark, A. E.; van Gunsteren, W. F. V. A biomolecular force field based on the free enthalpy of hydration and solvation: The GROMOS forcefield parameter sets 53A5 and 53A6. J. Comput. Chem. 2004, 25, 1656-1676.

[29] Berendsen, H. J. C.; Postma, J. P. M.; Di Nola, A.; Haak, J. R. L. Molecular dynamics with coupling to an external bath. J. Chem. Phys. 1984, 81, 3684-3690.

[30] Hess, B. A. P-LINCS: A parallel linear constraint solver for molecular simulation. J. Chem. Theor. Comput. 2008, 4, 116-122.

[31] Berendsen, H. J. C.; van der Spoel, D.; van Drunen, R. GROMACS-A message-passing parallel molecular dynamics implementation. Comput. Phys. Comm. 1995, 91, 43-56,

[32] Lindahl, E.; Hess, B.; van der Spoel, D. GROMACS 3.0: A package for molecular simulation and trajectory analysis, J. Mol. Model. 2001, 7, 306-317.

[33] Kasson, P. M.; Pande, V. S. Molecular dynamics simulation of lipid reorientation at bilayer edges. Biophys. J. 2004, 86, 3744-3749.

[34] Krishnamurty, S.; Stefanov, M.; Mineva, T.; Begu, S.; Devoisselle, J. M.; Goursot, A.; Zhu, R.; Salahub, D. R. Density functional theory-based conformational analysis of a phospholipid molecule (dimyristoylphosphatidylcholine). J. Phys. Chem. B 2008, 112, 1343313442.

[35] Wei, C. Radius and chirality dependent conformation of polymer molecule at nanotube interface. Nano Lett. 2006, 6, 1627-1631.

[36] De Vries, A. H.; Mark, A. E.; Marrink, S. J. Molecular dynamics simulation of the spontaneous formation of a small DPPC vesicle in water in atomistic detail. J. Am. Chem. Soc. 2004, 126, 4488-4489.

[37] De Vries, A. H.; Mark, A. E.; Marrink, S. J. Molecular dynamics simulation of the spontaneous formation of a small DPPC vesicle in water in atomistic detail. J. Am. Chem. Soc. 2004, 126, 4488-4489.

[38] Lu, J.; Xu, Y.; Chen, J.; Huang, F. Effect of lysophosphatidylcholine on behavior and structure of phosphatidylcholine liposomes. Sci. China Ser. C 1997, 40, 622-629. 